\title{
BPTF inhibits NK cell activity and the abundance of natural cytotoxicity receptor co-ligands
}

\author{
Kimberly Mayes ${ }^{1}$, Zeinab Elsayed ${ }^{1}$, Aiman Alhazmi', Michael Waters ${ }^{2}$, Suehyb G. \\ Alkhatib ${ }^{1}$, Mark Roberts ${ }^{1}$, Carolyn Song ${ }^{1}$, Kristen Peterson ${ }^{1}$, Vivian Chan ${ }^{1}$, Nikhil \\ Ailaney ${ }^{1}$, Pumoli Malapati ${ }^{1}$, Tana Blevins ${ }^{3}$, Berislav Lisnić ${ }^{4}$, Catherine I. Dumur ${ }^{3}$ \\ and Joseph W. Landry ${ }^{1}$

\footnotetext{
${ }^{1}$ The Department of Human and Molecular Genetics, Virginia Institute of Molecular Medicine, Massey Cancer Center, Virginia Commonwealth University, Richmond, Virginia 23298, USA

${ }^{2}$ The Department of Biochemistry, Virginia Commonwealth University, Richmond, Virginia 23298, USA

${ }^{3}$ The Department of Pathology, Virginia Commonwealth University, Richmond, Virginia 23298, USA

${ }^{4}$ The Center for Proteomics and Department for Histology and Embryology, University of Rijeka, Faculty of Medicine, 51000 Rijeka, Croatia
}

Correspondence to: Joseph W. Landry, email: joseph.landry@vcuhealth.org

Keywords: BPTF, chromatin remodeling, antitumor immunity, NK cell, heparanase

Received: January 31, $2017 \quad$ Accepted: April 26, $2017 \quad$ Published: May 12, 2017

Copyright: Mayes et al. This is an open-access article distributed under the terms of the Creative Commons Attribution License 3.0 (CC BY 3.0), which permits unrestricted use, distribution, and reproduction in any medium, provided the original author and source are credited.

\section{ABSTRACT}

Using syngeneic BALB/c mouse breast cancer models, we show that the chromatin remodeling subunit bromodomain PHD finger transcription factor (BPTF) suppresses natural killer (NK) cell antitumor activity in the tumor microenvironment (TME). In culture, BPTF suppresses direct natural cytotoxicity receptor (NCR) mediated NK cell cytolytic activity to mouse and human cancer cell lines, demonstrating conserved functions. Blocking mouse NCR1 in vivo rescues BPTF KD tumor weights, demonstrating its importance for the control of tumor growth. We discovered that BPTF occupies heparanase (Hpse) regulatory elements, activating its expression. Increased heparanase activity results in reduced cell surface abundance of the NCR co-ligands: heparan sulfate proteoglycans (HSPGs). Using gain and loss of function approaches we show that elevated heparanase levels suppress NK cell cytolytic activity to tumor cells in culture. These results suggest that BPTF activates heparanase expression, which in turn reduces cell surface HSPGs and NCR co-ligands, inhibiting NK cell activity. Furthermore, gene expression data from human breast cancer tumors shows that elevated BPTF expression correlates with reduced antitumor immune cell signatures, supporting conserved roles for BPTF in suppressing antitumor immunity. Conditional BPTF depletion in established mouse breast tumors enhances antitumor immunity, suggesting that inhibiting BPTF could provide a novel immunotherapy.

\section{INTRODUCTION}

To escape antitumor immunity tumor cells suppress natural killer (NK) cell activity [1]. Toward this end, tumor cells alter ligand presentation to NK cell receptors by acquiring genetic and epigenetic changes to the genome [2]. Understanding how epigenetics affects these changes is important because epigenetic modifications are reversible and, therefore, could be corrected with therapeutics [3].
One class of NK cell activating receptors are the natural cytotoxicity receptors (NCRs) [4]. There are 3 NCRs in humans (NKp30, NKp44, and NKp46) and 1 in mice (NCR1). These receptors can control tumor growth in vivo, and promote cytolytic activity to cancer cells in vitro [5-8]. Both human and mouse NCRs recognize heparan sulfate (HS) chains on cell surface heparan sulfate proteoglycans (HSPG) [9]. These HS chains bind growth factors, cytokines and proteins to regulate a variety of biological processes [10]. 
In mammals, HS are removed from HSPGs by heparanase to release bound factors and reorganize the extracellular matrix. In most normal cells HPSE expression is low, but it is commonly upregulated in many cancers to promote cell growth, motility, metastasis and inflammation [11].

One epigenetic regulator is the ATP-dependent chromatin remodeling complex, nucleosome remodeling factor (NURF). In mammals it is composed of 3 subunits: bromodomain PHD-finger containing transcription factor (BPTF), which is both essential and unique to NURF; the ISWI ATPase SNF2L; and the WD repeat protein pRBAP46/48 [12-14]. NURF slides nucleosomes in cis to alter accessibility of DNA for transcription factor binding, which ultimately regulates gene expression [12]. NURF is essential for embryonic development but is not cell essential $[15,16]$. The BPTF gene is frequently amplified and overexpressed in a variety of cancers including breast, lung, and brain [17], though how NURF functions in cancer biology is just beginning to be understood. To better understand how epigenetic regulators, and NURF in particular, influence tumor biology, we pursued a loss of function approach using well established syngeneic breast cancer models.

\section{RESULTS}

\section{NK cell-mediated antitumor immunity is enhanced to BPTF-depleted breast tumors}

To investigate roles for NURF in cancer cell biology, we transduced the well-established 67NR and $66 \mathrm{cl} 4$ mouse breast cancer cell lines [18] with retroviruses expressing control (Ctrl-sh1 or Ctrl-sh2) or BPTF shRNAs (Bptf-sh1 or Bptf-sh2) (Figure 1A). BPTF knockdown (KD) was used to deplete NURF because it is unique and essential to the complex [13, 14]. In culture we observed equivalent doubling times, cellular morphology, and levels of apoptosis (Supplementary Figure 1A-1C). To discover novel roles for BPTF in tumor biology, we transplanted the $66 \mathrm{cl} 4$ or $67 \mathrm{NR}$ lines into the $4^{\text {th }}$ mammary fat pad of syngeneic BALB/c mice. After 3-4 weeks, we observed reduced BPTF KD tumor weight (Figure 1B). Tumor weights were used instead of volume to measure growth because BPTF KD tumors grow flat, confounding volume-based comparisons to controls [19]. Microarray expression profiling of control and BPTF KD tumors discovered an enrichment of genes with gene ontology (GO) terms which included immune response descriptors (Supplementary Figure 2A; Supplementary Data Set 1). In agreement with microarray data, KEGG analysis of a combined gene list from both tumor types identified an abundance of genes involved in the immune response (Figure $1 \mathrm{C}$; for high resolution see Supplementary Figure 2B; Supplementary Data Set 1) [20]. To confirm the importance of the immune response for BPTF KD tumor growth, we repeated our tumor studies in an immune-deficient NOD/SCID, Ifrg2 $\mathrm{r}^{-/}$(NSG) background [21]. These experiments showed equivalent BPTF KD tumor weights to controls, demonstrating the immune system is required to reduce the growth of BPTF KD tumors (Figure 1D).

To identify immune cells that are important for reducing BPTF KD tumor growth, we repeated our tumor studies in mice depleted of NK cells, CD8+ T cells, or CD4+ T cells by monoclonal antibody $(\mathrm{mAb})$ treatments. We observed improved growth of 67NR and 66cl4 BPTF KD tumors with NK cell depletion (anti-asialo-GM1 $\mathrm{mAb}$ ), but not with $\mathrm{CD} 8+$ or CD4+ T-cell depletion, indicating that NK cells are required for reduced BPTF KD tumor growth (Figure 1E) (Supplementary Figure 3A-3C).

We next examined the abundance and activation of NK cells in the BPTF KD tumor microenvironment (TME)[22]. This analysis showed a greater population of NK cells (CD3-, NCR1+) in 67NR tumors compared to 66cl4 tumors (Figure 2A). While present, the CD3, NCR1+ populations did not change with BPTF KD in 66cl4 or 67NR TME (Figure 2B). However, we observed a greater percentage of $\mathrm{NCR} 1+, \mathrm{CD} 69^{\text {high }}$ cells in $67 \mathrm{NR}$ and $66 \mathrm{cl} 4 \mathrm{BPTF}$ KD tumors, demonstrating that a greater fraction of NK cells in BPTF KD tumors are activated as defined by CD69 expression (Figure 2C and 2D).

\section{NK cell cytotoxic activity is enhanced to BPTF depleted cancer cells in vitro}

Coculture of purified naive mouse NK cells with 67NR and $66 \mathrm{cl} 4$ cells showed enhanced activation and cytolytic activity to BPTF KD targets (Figure 3A) (Supplementary Figure 4A-4D). To determine if BPTF KD cells are more sensitive to NK cell-mediated killing, we stimulated their cytolytic activities independent of ligand using $\mathrm{PMA}+$ ionomycin $(\mathrm{P}+\mathrm{I}) . \mathrm{P}+\mathrm{I}$ activated $\mathrm{NK}$ cells killed control and BPTF KD targets equivalently, indicating that BPTF KD targets are not more sensitive to lysis by NK cells (Supplementary Figure 4E). To determine if soluble factors enhance NK cell activity, we pre-incubated naive mouse NK cells with media from cultured control or BPTF KD $67 \mathrm{NR}$ or $66 \mathrm{cl} 4$ tumor cells prior to their use in the cytolytic assay. From these experiments, we observed equivalent NK cell cytolytic activity toward BPTF KD targets with media preconditioned with either control or BPTF KD cells (Supplementary Figure 4F). These results in combination demonstrate that changes on the surface of BPTF KD tumor cells, as opposed to a soluble factor, improve NK cell activation and cytolytic activity.

We next determined if the enhanced NK cell cytolytic activity to BPTF KD targets is conserved between mice and humans. Toward this end, we cocultured the human NK-92 cell line [23] with either mouse $(67 \mathrm{NR}, 66 \mathrm{cl})$ ) or human (T47D, MDA-MB-436, SH-SY5Y) BPTF KD targets (Supplementary Figure 4G). From these experiments we 


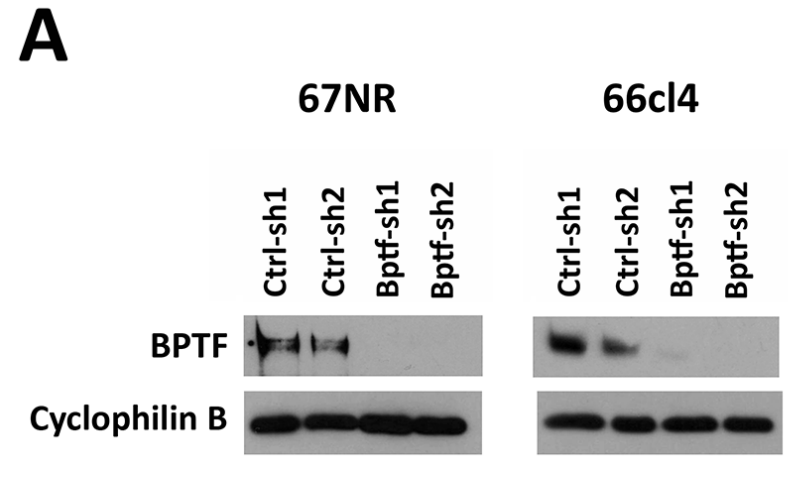

D

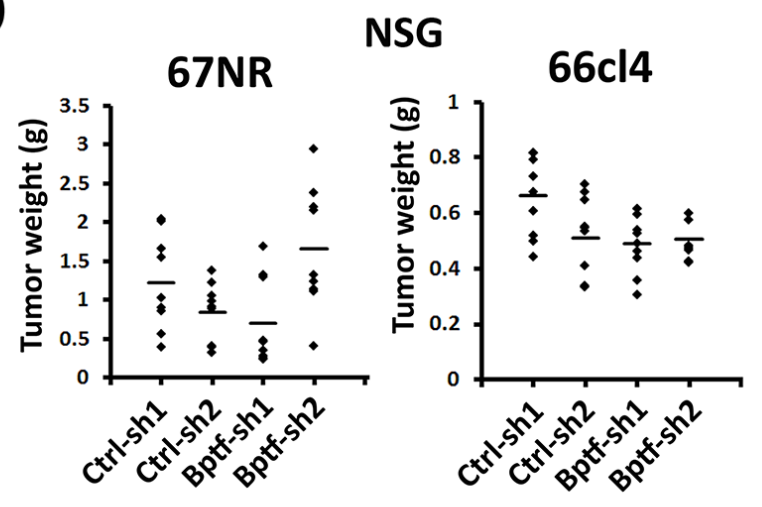

B

\section{BALB/c}
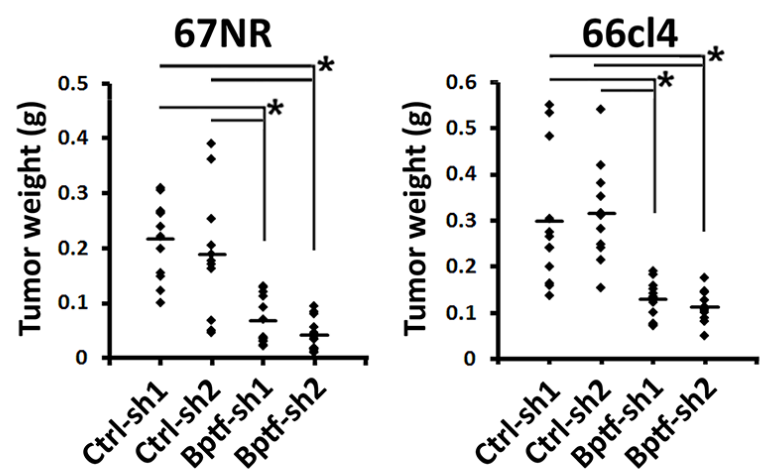

E
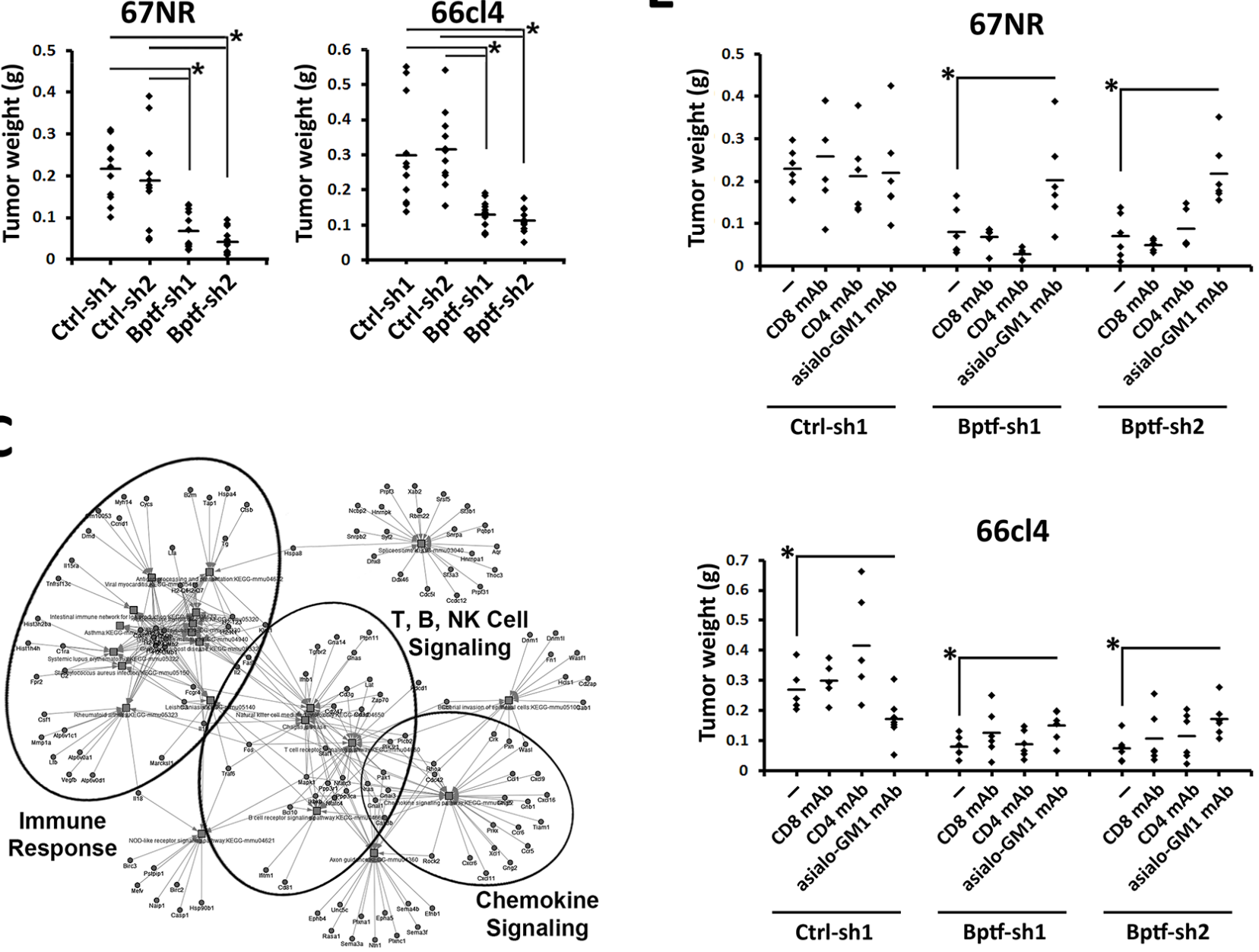

Figure 1: NK cells are required to reduce BPTF KD 67NR and 66cl4 tumor weight. (A) BPTF Western blot analysis from control (Ctrl-sh1, Ctrl-sh2) and BPTF KD (Bptf-sh1, Bptf-sh2) 67NR and 66cl4 total cell extracts. Cyclophilin B is used as a loading control. (B) $67 \mathrm{NR}$ and $66 \mathrm{cl} 4$ tumor weights harvested from BALB/c mice ( $\mathrm{n} \geq 11$ biological replicates, $*=$ ttest pvalue $<0.003$ ). (C) Low resolution KEGG pathway analysis of $67 \mathrm{NR}$ and $66 \mathrm{cl} 4$ significantly deregulated genes highlighting clusters of genes with function in the immune system (For high resolution please refer to Supplementary Figure 2B). (D) 67NR and 66cl4 tumor weights harvested from NSG mice ( $\mathrm{n}=9$ biological replicates). (E) 67NR and 66c14 tumor weights harvested from undepleted, or CD8, CD4 or asialo-GM1 mAb depleted BALB/c mice $(\mathrm{n}=6$ biological replicates, $*=$ ttest pvalue $<0.05)$. Some dots overlap. 
observed enhanced cytolytic activity to all BPTF KD cell lines, indicating that changes leading to enhanced cytolytic activity are conserved (Figure 3B and 3C).

A
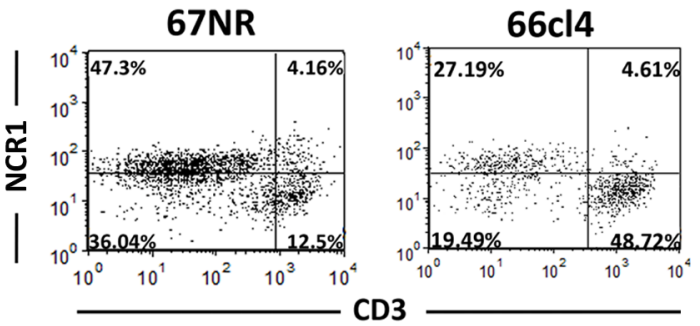

B
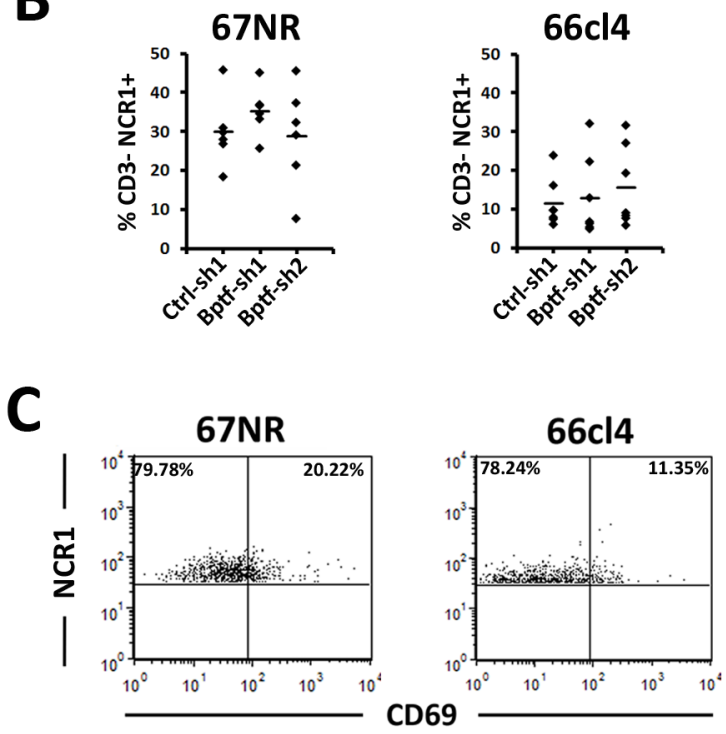

D
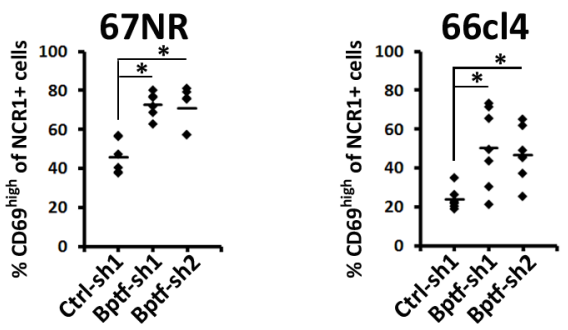

Figure 2: NK cells are more active in BPTF depleted tumor microenvironments. (A) Representative flow cytometry dot plots of live tumor infiltrating NK cells stained

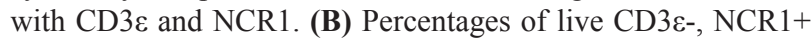
NK cells from $A$ ( $n \geq 6$ biological replicates). (C) Representative flow cytometry dot plots of live tumor infiltrating NK cells stained with CD69 and NCR1. CD69 $9^{\text {high }}$ is defined by CD69 staining of live CD3E- NCR 1+ cells from the spleen. (D) Percentages of live NCR $1+$ cells that are CD69high ( $\mathrm{n}=6$ biological replicates, * $=$ ttest pvalue $<0.006$ ). Quantitative data shown represent mean \pm stdev.

\section{BPTF regulates cell surface HSPG abundance and Hpse expression}

We conducted microarray gene expression analyses of control and BPTF KD 67NR and 66cl4 tumors from NSG mice to identify candidate BPTF-regulated NK cell receptor ligands. Analysis of these data sets focused on activating receptor ligands because they are conserved between mice and humans [24]. This is not true of the inhibitory MHC ligands which are species restricted in activity [25], and not significantly BPTF regulated in $67 \mathrm{NR}$ or $66 \mathrm{cl} 4$ cells (Supplementary Figure 5A). Combined qRT-PCR and microarray experiments identified three BPTF regulated genes with functions in HS metabolism ( $N d s t 1, N d s t 3$, Hpse) but did not identify any known activating NK cell receptor ligands (Figure 4A; Supplementary Figure 5B; Supplementary Data Set 1). HS and HSPGs are well characterized coligands for the NCRs, which are required for efficient NK cell-mediated tumor cell killing [26, 27].

Because HS is a known co-ligand to all NCRs, it is plausible that the enhanced NK cell cytolytic activity to BPTF KD cells occurs through the NCRs. mAb blocking of NKp30, but not NKp46 or NKG2D, on NK-92 cells inhibited the enhanced cytolytic activity against BPTF KD 66cl4 targets (Figure 4B). NKp44 was not included in our blocking studies because NK-92 cells do not express NKp44 [28]. NKp30 is highly expressed and is a major NCR used by NK-92 cells for antitumor activity [28, 29]. Functions for NKp30 in promoting NK cell activity to BPTF KD cells is conserved as demonstrated by reduced NK-92 cytolytic activity toward BPTF KD T47D targets with NKp30 blocking (Figure 4C). Similar reductions in mouse NK cell cytolytic activity was observed after NCR1 mAb blocking, the only NCR expressed in mouse NK cells (Figure 4D) [30]. Also, the addition of the NCR competitive inhibitor heparin [9] erased the NK-92 and mouse NK cell cytolytic activity against $67 \mathrm{NR}$ and $66 \mathrm{cl} 4$ BPTF KD targets (Supplementary Figure 5C and 5D).

We next used a recombinant NCR1-Ig fusion protein to measure the abundance of NCR1 ligands on the surface of BPTF KD 67NR and 66c14 cells. From these experiments, we discovered enhanced binding of NCR1-Ig to BPTF KD cells (Figure 4E and 4F). We then measured NCR1 activation using a cell line with an $I l-2$ reporter regulated by an NCR1- $\zeta$ fusion receptor [31]. Coculture experiments show elevated $I l-2$ gene expression when in contact with BPTF KD 67NR and 66cl4 cells (Figure 4G) (Supplementary Figure 5E), further suggesting that enhanced NK cell cytolytic activity results from increased NCR1 activity. We next used the NCR1 blocking mAb in tumor bearing mice and observed that BPTF KD tumor weights are rescued with NCR1 blocking (Figure 4H). As previously reported after NCR1 blocking mAb treatments NK cells are not depleted and cell surface NCR1 decreases (Supplementary Figure 5F and 5G)[32]. 
Our expression analysis identified Hpse, the major regulator of cell surface HS abundance, as a BPTFregulated gene in both $67 \mathrm{NR}$ and $66 \mathrm{cl} 4 \mathrm{BPTF} \mathrm{KD}$ tumors (Figure 4A)[33]. These results were confirmed in culture for the 67NR, 66cl4, T47D, SH-SY5Y, and MDA-MB-436 cell lines (Figure 5A) (Supplementary Figure 5H). In addition, an analysis of BPTF and HPSE expression in human cancers from the TCGA data sets revealed a highly significant correlation between high $B P T F$ expression and high HPSE expression in several cancer types including breast (see arrow) (Figure 5B). Consistent with Hpse gene expression, Western blotting discovered significant reductions in cell surface heparanase abundance with BPTF KD (Figure 5C). The importance of heparanase for enhanced NK cell activity to BPTF KD cells was investigated using bacterial heparinase treatments and heparanase KD cell lines. Pretreatment of tumor cells with bacterial heparinase suppressed the enhanced binding of NCR1-Ig to BPTF KD cells (Figure 5D) and reduced the enhanced cytolytic activity of NK cells to
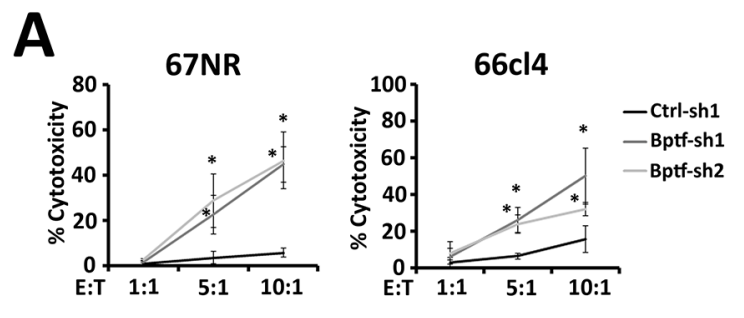

B

67NR
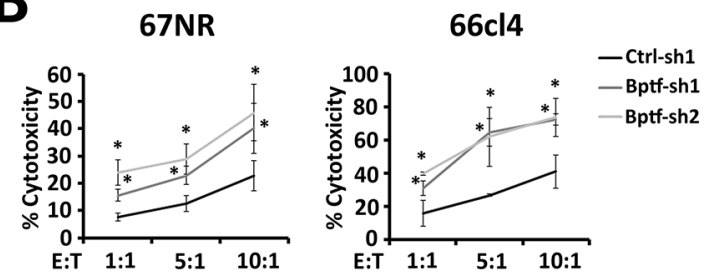

C
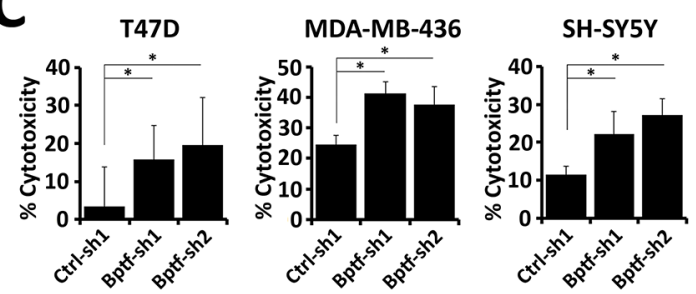

Figure 3: NK cells have greater cytolytic activity to BPTF KD targets. (A-C) Percent target cell cytolytic activity by LDH assay. (A) NK cells from naive BALB/c mice were cocultured with targets at the indicated effector:target $(\mathrm{E}: \mathrm{T})$ ratios ( $\mathrm{n}=3$ biological replicates, $*=$ ttest pvalue $<0.05$ ). (B) NK-92 cells were cocultured with targets at the indicated E:T ratios ( $\mathrm{n}=3$ biological replicates, $*=$ ttest pvalue $<0.05)$. (C) NK-92 cells were cocultured with human targets at a 10:1 E:T ratio $(\mathrm{n} \geq 3, *=$ ttest pvalue $<0.05)$. Quantitative data shown represent mean \pm stdev. 66cl4 BPTF KD targets (Supplementary Figure 5I). Similarly, we observed that heparanase KD in control $66 \mathrm{cl} 4$ cells results in enhanced NK cell cytolytic activity, consistent with heparanase abundance regulating NK cell activity (Supplementary Figure 5J and 5K). To determine if decreased Hpse expression with BPTF KD correlates with changes in cell surface HSPGs, we measured HSPG abundance by Western blotting cell surface protein extractions using an antibody to HS. Three HSPGs from these experiments had reproducibly increased levels in BPTF KD cells: a $\sim 150 \mathrm{kDa}$ band, a $\sim 55 \mathrm{kDa}$ band and $\mathrm{a} \sim 45 \mathrm{kDa}$ band (Figure 5E, see arrows). It is unlikely that expression of the HSPG core proteins are BPTFdependent because they were not BPTF-dependent from our microarray analyses (Supplementary Figure 5L). Additionally, only the MMP2 HSPG sheddase was deregulated in $67 \mathrm{NR}$, but not $66 \mathrm{cl} 4$, BPTF KD NSG tumors, suggesting that HSPG cell surface shedding is not BPTF-dependent (Supplementary Figure 5L). In addition, NURF occupies regulatory elements of Hpse because chromatin immunoprecipitation (ChIP) revealed BPTF occupancy broadly localized at the Hpse gene in 67NR and localized at the Hpse promoter in 66cl4 cells (Figure 5F) [34]. From these results, we propose a model where NURF activates Hpse in cancer cells, either directly or indirectly, elevating cell surface heparanase and reducing cell surface HSPGs. Because HSPGs are known co-ligands for NCRs, our model predicts that NURF suppresses NCR-mediated NK cytolytic activity by upregulating heparanase levels (Figure 5G).

\section{BPTF depletion enhances the immune response to established tumors}

To determine if BPTF suppression of the antitumor immune response could be conserved in human tumors, we monitored the immune cell composition from human breast tumor microarray data sets using the CIBERSORT algorithm [35]. From this analysis, we observed an enrichment of $\gamma \delta \mathrm{T}$ cells, resting CD4 T cells and B cells in tumors with high BPTF expression. Conversely, tumors expressing low levels of BPTF are enriched for active dendritic cells, macrophages, neutrophils, CD8+ $\mathrm{T}$ cells and Treg cells (Figure 6A). These results suggest that low BPTF levels correlate with increased abundance and activity of immune cells in human breast tumors. These results are supported by an independent analysis of the larger breast cancer provisional TCGA RNASeq data set [36]. This analysis revealed an increase in the expression of CD8 T cell, NK cell, macrophage and Treg cell markers, and immune cell cytokines in breast tumors with low levels of BPTF expression(Figure 6B) (Supplementary Figure 6A). We observe increased $N K p 44$ (NCR3) expression but not NKp46 (NCR1) possibly because the elevated IL2 expression in BPTF low tumors could downregulate $N K p 46$ [37]. 
To further test this model, we injected established $66 \mathrm{cl} 4$ and 4T1 tumors with replication-deficient adenovirus (rAd) expressing control or BPTF KD shRNA. 67NR tumors could not be used for these experiments because they undergo apoptosis after transduction by rAd in culture (data not shown). We had previously shown that BPTF KD 4T1 tumors upregulate antigen processing genes to improve the CD8+ T cell antitumor response [19]. Western blot analysis of $66 \mathrm{cl} 4$ and $4 \mathrm{~T} 1$ treated tumors show that $\mathrm{rAd}$ treatments resulted in BPTF KD (Figure 6C). BPTF KD in established tumors significantly reduced tumor weights compared to controls (Figure 6D), and we occasionally observed complete 66cl4 tumor regression with BPTF KD (rAd BPTF shRNA $=3$ of 17 treated tumors, rAd control shRNA $=0$ of 16 treated tumors). To measure the effects of BPTF KD on the antitumor immune response, we monitored the activation of $\mathrm{NK}$ and $\mathrm{CD} 8+\mathrm{T}$ cells in $66 \mathrm{cl} 4$ and 4T1 tumors, respectively. Consistent with our previous studies, BPTF KD in established tumors resulted in a greater percentage of CD69 $9^{\text {high }} \mathrm{NCR} 1+\mathrm{NK}$ cells in the 66cl4 TME, or CD69 ${ }^{\text {high }}$ CD8+ T cells in the 4T1 TME (Figure 6E). Increased activation of $\mathrm{NK}$ and $\mathrm{CD} 8+\mathrm{T}$ cells coincided with elevated IFN $\gamma$, suggesting a conversion to a Th1 TME (Figure 6F). As a control, transduction of NK-92 cells and splenic mouse OT1 T cells in culture with rAd to achieve BPTF KD followed by coculture assays show that BPTF KD does not significantly alter NK or T cell cytolytic activity (Supplementary Figure 6B-6F). These results indicate that these effector cells are likely functional after BPTF KD rAd treatments.

\section{DISCUSSION}

Understanding how tumors escape the immune response is of great interest to tumor immunologists. Epigenetic escape mechanisms, most prominently DNA methylation and histone deacetylation, suppress tumor cell antigenicity to inhibit $\mathrm{T}$ cells, and ligand expression to
A

\begin{tabular}{l|cc|cc|}
\cline { 2 - 5 } \multicolumn{1}{c|}{} & \multicolumn{2}{c|}{ 67NR } & \multicolumn{2}{c|}{ 66cl4 } \\
\hline NK Receptor & Gene & Fold Change & \multicolumn{2}{c|}{ Gene Fold Change } \\
\hline NCR1 & Ndst3 & -2.2 & Ndst1 & -1.5 \\
& Hpse & -3.3 & Hpse & -1.4 \\
NKG2D & - & - & - & - \\
2B4 & - & - & - & - \\
DNAM1 & - & - & - & - \\
LFA1 & - & - & - & - \\
CD16 & - & - & - & - \\
\hline
\end{tabular}

B

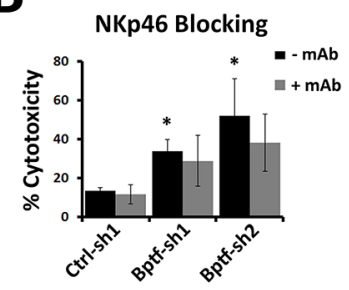

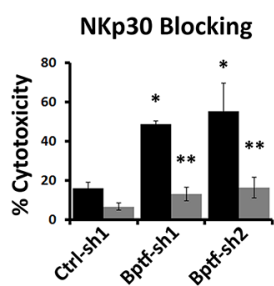

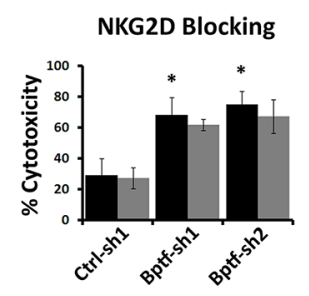

E

E
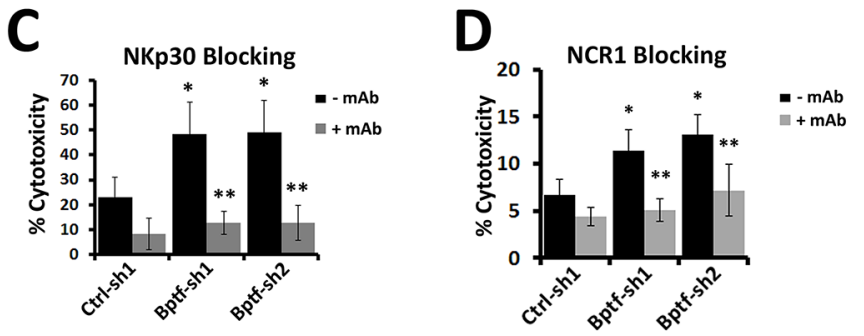

67NR

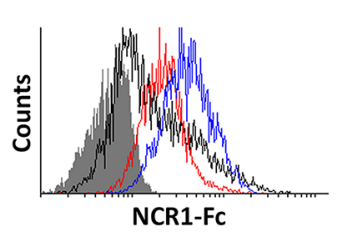

$66 \mathrm{cl} 4$

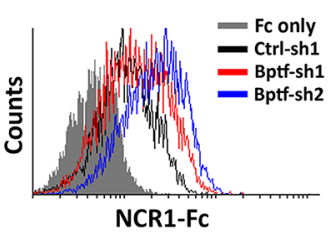

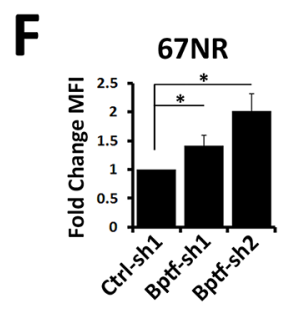
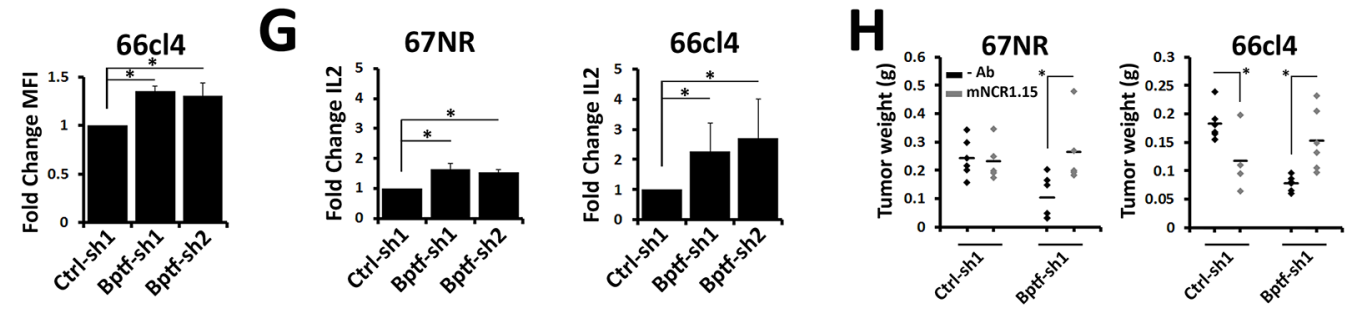

Figure 4: NK cell cytolytic activity to BPTF KD cells requires NCRs. (A) Changes in NK receptor ligand expression measured by microarray in BPTF KD tumors harvested from NSG mice compared to controls. Hpse was measured independently by qRT-PCR because it was not on the microarray $(*=$ ttest pvalue $<0.00002)$. (B-D) Percent target cell cytolytic activity by LDH assay. (B) NK-92 cells pretreated with anti-NKp46, anti-NKp30 or anti-NKG2D mAb were cocultured on 66c14 targets at a 10:1 E:T ratio (n=3 biological replicates, $*=$ ttest pvalue $<0.05)$. (C) NK-92 cells pretreated with anti-NKp30 were cocultured with T47D targets at a 10:1 E:T ration (n $=3$ biological replicates, $*=$ ttest pvalue $<0.05)$. (D) Mouse NK cells pretreated with anti-NCR1 blocking mAb (clone mNCR1.15) were cocultured with $66 \mathrm{cl} 4$ targets at a 5:1 E:T ratio $(\mathrm{n}=3$ biological replicates, $*=$ ttest pvalue $<0.04)$. $(*=$ significant to no $\mathrm{mAb}$ Ctrl-sh1, $* *$ = significant to the respective no mAb hairpin). (E) Representative flow cytometry histograms of NCR1-Ig binding to 67NR and 66cl4. (F) Fold change of MFI from D ( $\mathrm{n}=3$ biological replicates, ${ }^{*}=$ ttest pvalue $\left.<0.03\right)$. (G) qRT-PCR analysis of $I l-2$ expression in Ncr1- $\zeta, I l-2$ reporter BW cells incubated with control or BPTF KD cells for $48 \mathrm{hrs}(\mathrm{n} \geq 3$ biological replicates, $*=$ ttest pvalue $<0.05)$. (H) $67 \mathrm{NR}$ and $66 \mathrm{cl} 4$ tumor weights harvested from untreated or mNCR1.15 treated BALB/c mice $(\mathrm{n} \geq 4$ biological replicates, $*=$ ttest pvalue $<0.05)$. All quantitative data shown represent mean \pm stdev. 
A

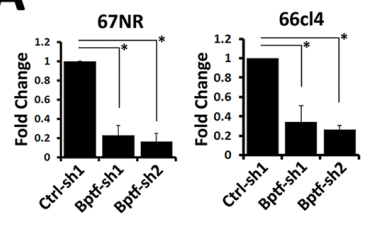

C

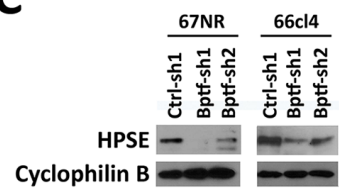

B

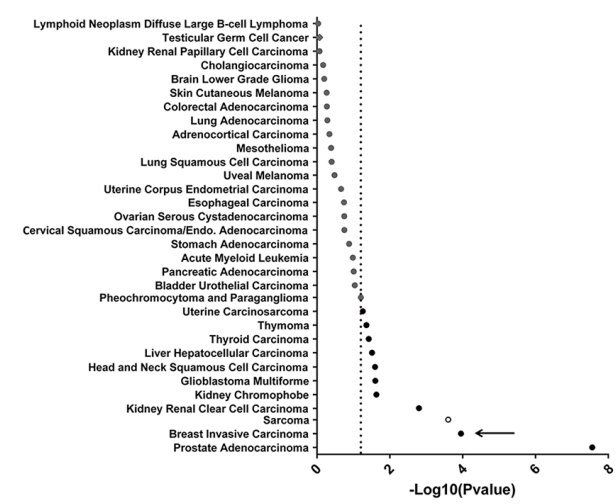

$\mathbf{F}$

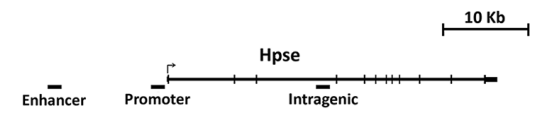

$\mathbf{E}$
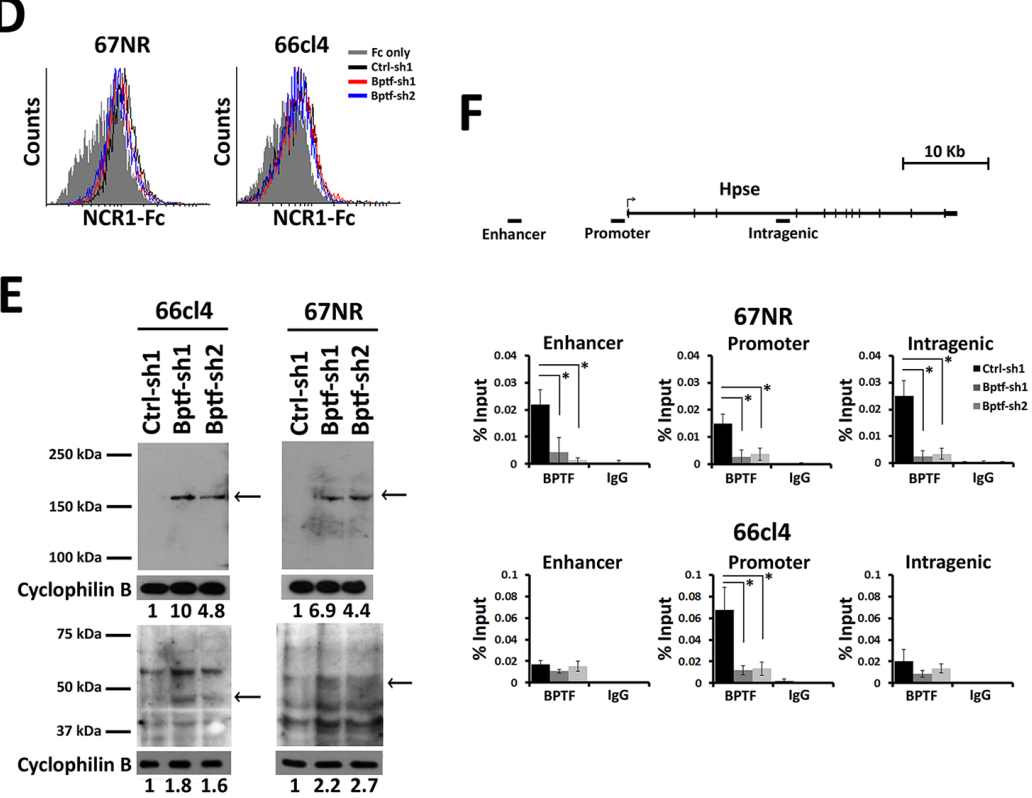

$66 \mathrm{cl} 4$

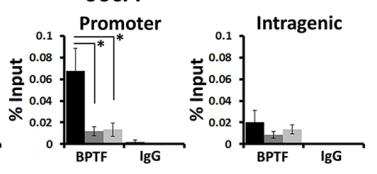

G
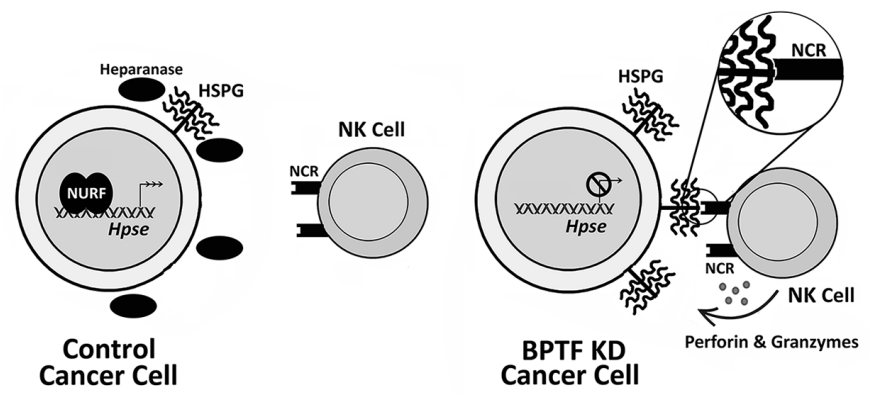

Figure 5: BPTF regulates Hpse expression. (A) qRT-PCR analysis of Hpse expression from $67 \mathrm{NR}$ and $66 \mathrm{cl} 4 \mathrm{cells}$ ( $\mathrm{n}=3 \mathrm{biological}$ replicates, ${ }^{*}=$ ttest pvalue $<0.006$ ). (B) Differential Hpse expression in BPTF high and BPTF low expression groups from TCGA datasets. The negative base $10 \log$ of the p-value for each t-test is shown. Dashed line: significance cutoff pvalue $=0.05$. Filled circles: positive correlation. Outlined circles: negative correlation. Breast cancer data set is shown with arrow. (C) Western blot analysis of cell surface HPSE from control and BPTF KD 67NR and 66cl4 cells. Cyclophilin B was used as a normalization control. (D) Representative flow cytometry histogram of NCR1-Ig binding to bacterial heparinase treated tumor cells. (E) Western blot analysis of cell surface HSPG using anti-HS primary mAb. Cyclophilin B was used as a normalization control. Arrows: reproducible changes in HSPG abundance with BPTF KD. ImageJ relative quantitation to controls are shown as numbers below blots. (F) BPTF ChIP at mouse Hpse in control and BPTF KD $67 \mathrm{NR}$ and $66 \mathrm{cl} 4$ cell lines $(\mathrm{n}=3$ biological replicates, $*=$ ttest pvalue $<0.04)$. (G) A Model: In tumor cells NURF stimulates Hpse expression, and as a result cell surface heparanase abundance. Increased heparanase abundance reduces cell surface HSPGs and NCR HS co-ligand abundance, inhibiting NK-cell antitumor activity. When NURF is depleted, Hpse expression and heparanase is reduced. Reduced heparanase abundance increases cell surface HSPGs and NCR HS co-ligands, improving NK cell-mediated antitumor activity. All quantitative data shown represent mean \pm stdev. 

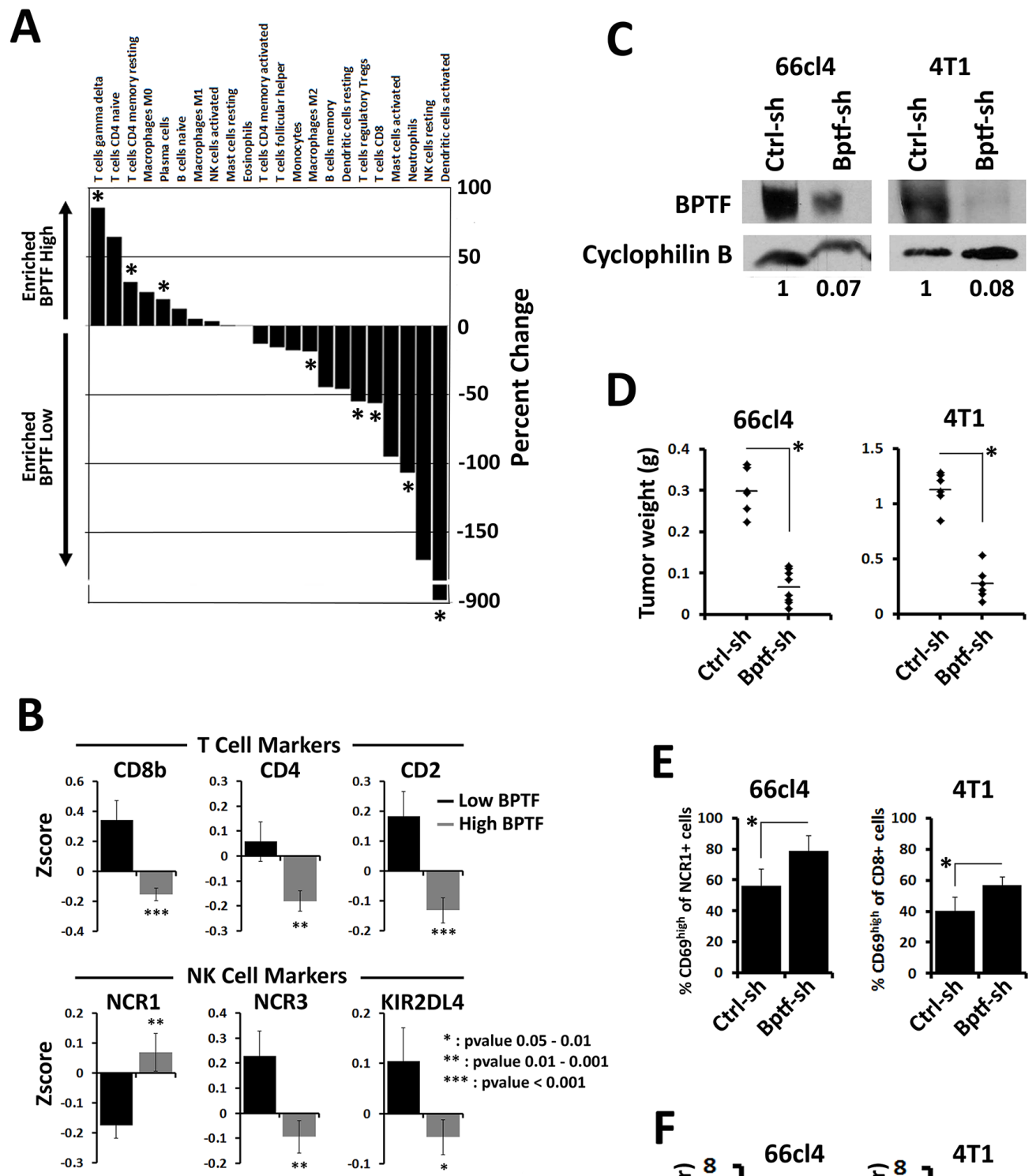

\section{NK Cell Markers}

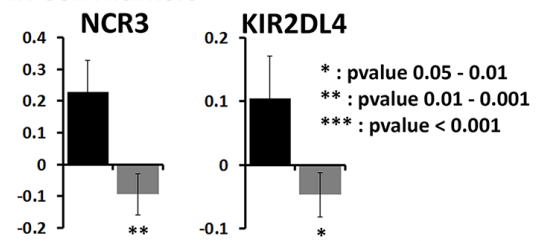

$\mathbf{E}$
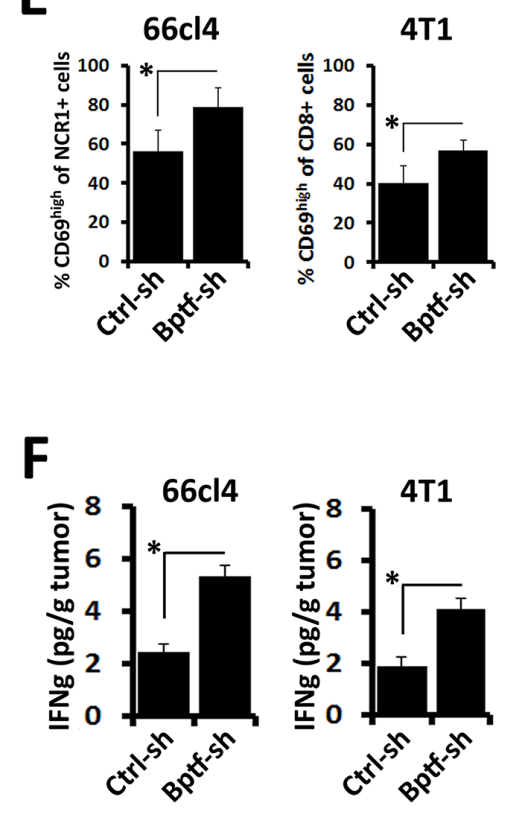

Figure 6: BPTF is required for an immune suppressive tumor microenvironment. (A) Differential immune profiles identified using CIBERSORT between $B P T F$ high and $B P T F$ low expression groups from microarray expression profiles $(*=$ ttest pvalue $<0.1)$. (B) Differential expression of immune cell markers in BPTF high and BPTF low expression groups from Zscore normalized expression data from breast cancer TCGA datasets (pvalues are shown in panel). (C) BPTF Western blot analysis of 66c14 and 4T1 tumors injected with rAd expressing either control or BPTF KD shRNAs. Cyclophilin B was used as a loading control. ImageJ relative quantitation to controls are shown as numbers below blots. (D) Weights of 66cl4 and 4T1 tumors after treatment with $\mathrm{rAd}(\mathrm{n} \geq 6$ biological replicates, * $=$ ttest pvalue $<0.000003$ ). (E) Flow cytometry analysis of 66c14 tumor infiltrating NK cells and 4T1 tumor infiltrating CD8+ T cells. Active lymphocytes are quantified as percent CD69high of total infiltrating NK cells (NCR1+) or CD8+ T cells $(\mathrm{CD} 8+)(\mathrm{n} \geq 4$ biological replicates, * $=$ ttest pvalue $<0.01$ ). (F) IFN $\gamma$ concentrations from $66 \mathrm{cl} 4$ and 4T1 tumor extracts measured by ELISA ( $\mathrm{n} \geq 6$ biological replicates, $*=$ ttest pvalue $<0.04)$. All quantitative data shown represent mean \pm stdev. 
inhibit NK cells [38]. Because many epigenetic regulators are required for gene expression, chromatin remodeling is predicted to have similar functions in tumor cells. Using transplantable tumor models, we tested this prediction and discovered that BPTF, and by extension NURF, represses NK-mediated antitumor activity. We also observed protumor NK cell activities to 66cl4 tumors, which the use of a NCR1 blocking mAb suggest are NCR1 mediated. NCR1 expressing NK cells and immature lymphoid cells can control the immune response by direct killing of dendritic cells and T cells $[39,40]$, which could suppress an adaptive immune response to $66 \mathrm{cl} 4$ tumors, possibly explaining their pro-tumor activities. In total, these results support the idea that BPTF is a novel regulator of the NK cell-mediated antitumor immune response.

To identify BPTF-dependent NK receptor ligands, we used two complementary approaches. We first used the human NK-92 cell line to demonstrate that the BPTFregulated ligand(s) are conserved across species, and second used microarrays to discover that components of the HS synthesis and degradation pathways are BPTF-dependent in tumors. The most noteworthy BPTF-regulated factor discovered was the HS degrading enzyme heparanase, an enzyme which is highly conserved in both sequence and function between mouse and humans [41]. NURF could directly regulate Hpse expression because BPTF occupies its regulatory elements, but it is also equally possible that NURF regulates Hpse indirectly. Characterizing NURFregulated chromatin remodeling activities and mechanisms of NURF recruitment to these regulatory elements would be required to support a model where NURF directly regulates Hpse. Using similar approaches we discovered that NURF directly regulates chromatin structures surrounding CTCF binding sites to influence their regulatory activity [15]. One possibility is that NURF could be recruited to the Hpse promoter through its ability to bind the promoter associated histone modifications $\mathrm{H} 3 \mathrm{~K} 4 \mathrm{me} 3$ and $\mathrm{H} 4 \mathrm{~K} 16 \mathrm{ac}$ [42]. Alternatively, NURF could be recruited to Hpse regulatory elements through interactions with novel transcription factors [12].

HS and HSPGs are highly conserved, common coligands to all NCRs, and they influence NK cell-mediated antitumor immunity in both humans and mice $[5,9$, 11]. Consistent with BPTF regulating the expression of NCR co-ligands, NKp30 and NCR1 mAb blocking and heparin inhibition experiments reduce the enhanced NK cell cytolytic activity to BPTF KD targets in vitro. Also consistent, we observe rescue of BPTF KD tumor growth when NCR1 blocking $\mathrm{mAb}$ are used in vivo and is consistent with other reports that NCR1 is important for antitumor activity $[5,43,44]$. Coincident with reduced cell surface heparanase, Western blotting identified HSPGs which increase in abundance on the surface of BPTF KD cells. Heparanase binds HSPGs, stimulating endocytosis and eventually HS cleavage, which could explain the observed increased cell surface HSPG levels on BPTF KD cells [41]. These results in total suggest a model where BPTF upregulates heparanase expression to down regulate cell surface HSPG levels, a co-ligand to NCR1, reducing NK cell cytolytic activity. While this model is consistent with the data we cannot rule out that bonified, and yet unidentified, NCR ligands are NURF regulated which contribute to changes in NK cell activity [45].

The significance of BPTF, and by extension NURF, regulation of HPSE in cancer could have broader implications. HPSE is commonly upregulated in cancer cells to impact angiogenesis, metastasis, tumor growth and inflammation [11]. It is therefore plausible that differences in tumor shape with BPTF KD could result from abnormal angiogenesis, or establishing a fibrin shell. Decreases in heparanase levels could also contribute to defects in metastases, as previously observed with BPTF KD melanoma tumors [46]. Defects could result from reductions in cell motility [45], or from enhancements of NK cell antitumor activity which is most significant to metastatic cancer cells [47]. These hypotheses, partially supported by our data, warrant more detailed studies on roles for BPTF during metastasis.

NK cells have greatest therapeutic significance to hematological malignancies, where NK cell activity can result in disease remission. NK cell activity against solid tumors is less significant because the immune suppressive TME inhibits NK cell infiltration and activity [48]. We show for the first time that depleting BPTF in the TME could circumvent problems inherent to the use of NK cells as an immunotherapy toward solid tumors. BPTF depletion in $66 \mathrm{cl} 4$ tumors with rAd resulted in tumor regression with about $18 \%$ frequency, despite incomplete BPTF KD. Regression could result from the elevated IFN $\gamma$ converting the TME to a Th1 microenvironment and enhancing the activity of other effector cells, or from promoting epitope spreading, which could expand novel tumor reactive T cell clones [49]. We speculate that tumor regression is not observed in the 4T1 model because of significant immunosuppressive activity of the remaining myeloid derived suppressor cells after gemcitabine treatments [50]. It is also noted that there are observed differences in the immune response between mouse and human BPTF KD vs BPTF low tumors. Using the mouse tumor models we do not detect significant differences in NK cell abundance with BPTF KD, but differences in abundance are observed in human breast tumors with low BPTF expression. These differences could be due to differences in how mouse tumors or NK cells respond to low intratumor BPTF levels compared to similar conditions in humans. Alternatively, increased infiltration of NK cells in BPTF low human breast tumors could result from increased CD8 T cell antitumor activity, which we previously observed to the 4T1 mouse breast tumor model [19]. To resolve these unknowns, expanded studies on the response of human tumors to BPTF inhibition will need to be conducted, which was limited in the current study to 
the use of cancer cell lines in vitro. It is our ultimate goal to translate these findings into a novel immunotherapy, which could be initiated by using newly identified BPTF bromodomain small molecule inhibitors [51] alone or in combination with established immune stimulating chemotherapies [52] or immunotherapies like NK cell adoptive cell transfer [53].

\section{MATERIALS AND METHODS}

\section{Mice}

$\mathrm{BALB} / \mathrm{cJ}, \underline{\mathrm{NOD}} / \underline{\mathrm{SC}} \mathrm{CD} / \mathrm{Ifrg} 2 \mathrm{r}^{-/}$(NSG) and C57BL/6$\mathrm{Tg}$ (TcraTcrb)1100Mjb/J (OT1) female mice 6-8 weeks of age weighting $\sim 20 \mathrm{~g}$ (Jackson Laboratory) were housed under aseptic barrier conditions as approved by Virginia Commonwealth University IACUC.

\section{Cell culture}

67NR, 66cl4, 4T1 (Wayne State University, 2010) SY5Y and HEK 293T (ATCC, 2010) were cultured in complete media (CM) (DMEM, 10\% FBS, 1\% NEAA, $1 \%$ glutamine, and $1 \%$ penicillin/streptomycin). T47D and MDA-MB-436 (ATCC, 2010) were cultured in RPMI, 10\% FBS, 1\% NEAA, 1\% glutamine, 1\% penicillin/streptomycin and $5 \mu \mathrm{g} / \mathrm{ml}$ insulin. NK-92 cells (ATCC, 2012) were cultured in Alpha MEM, $12.5 \%$ horse serum, $12.5 \%$ FBS, $2 \mathrm{mM}$ L-glutamine, $1.5 \mathrm{~g} / \mathrm{L}$ sodium bicarbonate, $0.2 \mathrm{mM}$ inositol, $0.1 \mathrm{mM}$ 2-mercaptoethanol, $0.02 \mathrm{mM}$ folic acid and $200 \mathrm{U} / \mathrm{ml} \mathrm{Il-2.}$ OT1 T cells were cultured in CM with 1\% HEPES, $5 \times 10$ ${ }^{5} \mathrm{M}$ 2-mercaptoethanol and $500 \mathrm{U} / \mathrm{ml}$ mouse Il-2. Cell lines were validated by Wayne State University or ATCC prior to shipment by short tandem repeat (STR) profiling. Mycoplasma contamination was tested and confirmed to be negative every 2 years using Universal Mycoplasma Detection Kit (ATCC).

ShRNAs were introduced into $67 \mathrm{NR}$ and $66 \mathrm{cl} 4$ cells using the pSIREN-RetroQ system (Clonetech). pSIREN plasmids Ctrl-sh1, Ctrl-sh2, Bptf-sh1, Bptf-sh2, HPSEsh1, HPSE-sh2 are available at Addgene as stock numbers 73665, 73666, 73667, 83045, 92033, 92034 respectively. ShRNAs were introduced into T47D, MDA-MB-436 and SH-SY5Y cells using the lentiviral pLVTHM system. pLVTHM plasmids Ctrl-sh1, Bptf-sh1, Bptf-sh2 are available at Addgene as stock numbers 83046, 83277, 83278 , respectively. Transduced cells were selected with $0.5 \mu \mathrm{g} / \mathrm{ml}$ puromycin after 48 hours.

\section{Replication-deficient adenovirus}

To construct rAd-BPTF and rAd-Luc, we used the AdenoQuick cloning system (OD260, Inc.) according to the manufacturer's instructions. pAD388 cosmids Ctrl-sh,
Bptf-sh are available at Addgene as stock numbers 83275, 83276, respectively. The cosmid DNA was transfected into HEK-293T cells and serially amplified 3 times to obtain high titer virus [54]. Virus titer was determined by TCID50 according to the manufacturer's instructions. 66c14 and 4T1 cells were infected with $1 \times 10^{6}$ PFU rAd twice during a one week period.

Spleens from OT1 mice were harvested and depleted of erythrocytes with RBC lysis buffer and cocultured with mitomycin C-treated B16F10-OVA cells for 3 weeks. $3 \times 10^{6}$ cultured NK-92 or $5 \times 10^{5}$ expanded OT1 T cells were then infected with $2 \times 10^{7}$ or $2 \times 10^{6} \mathrm{rAd}$ in a volume of $10 \mathrm{ml}$ or $1 \mathrm{ml}$ for 5 days.

\section{Tumor studies}

$1 \times 10^{5} 67 \mathrm{NR}$ cells and $1 \times 10^{4} 66 \mathrm{cl} 4$ cells were injected into the fourth mammary fat pad of BALB/c or NSG mice. Tumors were analyzed at 21 days (67NR) or 28 days (66cl4). For rAd studies, BALB/c mice were inoculated with $1 \times 10^{6} 66 \mathrm{cl} 4$ or $3 \times 10^{5} 4 \mathrm{~T} 1$ cells and injected intratumorally with rAd-Luc or rAd-BPTF $\left(1 \times 10^{9} \mathrm{PFU}\right.$ in 100ul) every 3 days for 3 weeks once tumors were $\sim 5 \mathrm{~mm}$ in any dimension. Animals were euthanized 1 week after the last rAd injection. For 4T1 studies, mice were injected intraperitoneally (I.P.) with $1.2 \mathrm{mg} /$ mouse gemcitabine 5 days after tumor inoculation and once every 7 days thereafter.

\section{mAb depletions}

GK1.5, 2.43 and SH-34 mAb depletions were performed as described previously [19]. Briefly, mAbs were purified from ascites fluid by ammonium sulfate fractionation and $225 \mu \mathrm{g} /$ mouse was injected on day -2 , and -1 . Tumors were inoculated on day 0 and $\mathrm{mAb}$ was injected once every 5 days following tumor inoculation.

\section{mNCR1.15 blockade}

$100 \mu \mathrm{g} /$ mouse mNCR1.15 (Center for Proteomics, University of Rijeka, Croatia) was injected on day -2 and -1. Cell lines were inoculated on day 0 and mNCR1.15 was injected once every 5 days thereafter.

\section{Western blot}

For total extracts, protein from cell cultures or homogenized tumors was extracted by TRI Reagent. Cell surface protein extraction was performed as described previously [55]. Primary antibodies: anti-BPTF (Millipore Cat\# ABE024), anti-HS (Millipore Cat\# MAB2040), and anti-HPSE (Santa Cruz Cat\# sc25826) then anti-mouse or rabbit HRP secondary (Cell Signaling). Loading was determined Cyclophilin B. 


\section{NK cell cytotoxicity assay}

NK cells were purified from the spleen of naïve BALB/c mice by negative selection using MACS separation (Miltenyi Biotech). Purified mouse NK cells or cultured NK-92 cells were placed on mitomycin $\mathrm{C}$-treated targets for $24 \mathrm{hrs}$ (mouse NK cells with mouse targets), 7 hrs (NK-92 cells with mouse targets) or 4 hrs (NK-92 cells on human targets). For antibody blocking experiments, NK cells were pre-incubated for $1 \mathrm{hr}$ with $10 \mu \mathrm{g} / \mathrm{ml}$ blocking antibodies to NCR1 (mNCR1.15 from Stipan Jonjic, Center for Proteomics, University of Rijeka, Croatia), hNKp46 (Biolegend Cat\# 13614), hNKp30 (Biolegend Cat\# 325204) or hNKG2D (BD Pharmingen Cat\# 552866). For hyperactivation, cocultured mouse NK cells were treated with $0.8 \mu \mathrm{M}$ PMA, $0.35 \mu \mathrm{M}$ Ionomycin and $50 \mathrm{U} / \mathrm{ml} \mathrm{IL-2.} \mathrm{For} \mathrm{media} \mathrm{preconditioning,} \mathrm{mitomycin}$ $\mathrm{C}$ treated targets were incubated for $24 \mathrm{hrs}$, growth media was then removed and incubated with NK cells for 24 hrs before assay on targets. For heparin competition experiments, cocultures were incubated with $100 \mu \mathrm{g} /$ $\mathrm{ml}$ heparin (Sigma) in growth media during coculture with target cells. For heparinase treatment, 66cl4 cells were pretreated with $1 \mathrm{U} / \mathrm{ml}$ bacterial heparinase I/III (Sigma) in DMEM, $1 \%$ BSA for one $\mathrm{hr}$ at $37^{\circ} \mathrm{C}$ before coculture with NK cells. Cell death was measured using the CytoTox 96® Non-Radioactive Cytotoxicity Assay (Promega).

\section{qRT-PCR}

Quantitative RT-PCR was performed as described previously [19]. Primer pairs are found in Supplementary Data Set 2.

\section{Flow cytometry}

Cultured 67NR and 66cl4 cells were stained with H2-Kd (Cat\# 553566), H2-Dd (Cat\# 558917), H2-Ad (Cat\# 553548), Qa1 (Cat\# 559829) antibodies or Annexin $\mathrm{V}$ (Cat\# 556421), and 7AAD viability dye. To measure NK activity, mouse NK cells were incubated for 24 hours with mitomycin $\mathrm{C}$ treated targets, removed and stained with NCR1 (Cat\# 137605) (Biolegend) and CD69 (Cat\# 561932) antibodies and 7AAD viability dye. To measure NK cells in the spleen, splenocytes were stained with CD3 and DX5 (Cat\#561066) antibodies and 7AAD viability dye.

Tumor infiltrating lymphocytes were purified as described previously [19]. Purified lymphocytes were stained with NCR1, CD69, CD3 (Cat\# 553060), or CD8 (Cat\# 100706) antibodies and 7AAD viability dye. All antibodies used for flow cytometry are from BD Pharmingen, unless otherwise specified.

The NCR1-Ig fusion protein (gift from Ofer Mandelboim from The Hebrew University of Jerusalem) was purified from HEK293T cells as described previously [56]. For heparinase treatment, $67 \mathrm{NR}$ and $66 \mathrm{cl} 4$ cells were treated with $10 \mathrm{U} / \mathrm{ml}$ bacterial heparinase I/III (Sigma) in $1 \%$ BSA DMEM for $1 \mathrm{hr}$ at $37^{\circ} \mathrm{C}$. Then $1 \times 10^{5}$ cells were incubated with $1 \mu \mathrm{g}$ NCR1-Ig for $1 \mathrm{hr}$ at $4^{\circ} \mathrm{C}$ and stained with PE- anti-human-IgG Fc (Biolegend Cat\# 409304).

\section{Chromatin immunoprecipitation (ChIP)}

ChIP was performed as previously described [15]. Primer pairs are found in Supplementary Data Set 2.

\section{Enzyme linked immunosorbent assay (ELISA)}

Tumors were minced and suspended in hanks balanced salts supplemented with complete protease inhibitor. After 3 cycles of freeze thaw, samples were centrifuged at $15,000 \mathrm{x} \mathrm{rcf}$ at $4^{\circ} \mathrm{C}$ for $15 \mathrm{~min}$ and the supernatant was collected. ELISA for IFN $\gamma$ (R\&D Systems) was performed according to the manufacturer's instructions.

\section{NCR1 reporter assay}

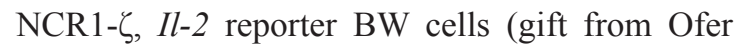
Mandelboim from The Hebrew University of Jerusalem) were cocultured with $67 \mathrm{NR}$ or $66 \mathrm{cl} 4$ targets at 1:1 E:T ratio for $48 \mathrm{hrs}$. Il2 expression was measured by qRT-PCR and normalized to NCR1- $\zeta$ expression. Primer pairs are found in Supplementary Data Set 2.

\section{Microarray}

RNA extraction, microarray analysis and statistical analysis were performed as described [57, 58]. Data is available as GEO accession \#GSE785756.

\section{Bioinformatics}

RNAseq Zscores were downloaded from The Cancer Genome Atlas (TCGA). For each cancer, patients were divided into high $(\mathrm{n}=325)$ and low $(\mathrm{n}=169)$ $B P T F$ expressing groups using a Zscore cutoff of $>1$ and $<-1$ respectively. A t-test was performed to evaluate differential expression of HPSE between the BPTF high and BPTF low groups. For immune cell markers, an unpaired t-test was performed to assess differential expression of immune cell markers between BPTF low and $B P T F$ high samples.

Microarray expression profiles from patients with breast cancer were downloaded from NCBI GEO (GSE2109) $(\mathrm{n}=348)$. Patients expressing 1 standard deviation above or below the mean level of expression were binned as BPTF high $(\mathrm{n}=40)$ and BPTF low (n $=45$ ), respectively. Probe level data from both groups were used as input for the CIBERSORT deconvolution algorithm. Immune profiles from $B P T F$ high and $B P T F$ low groups were compared. 


\section{Statistics}

Excel was used to calculate statistical differences between groups using the two-tailed Student's t-test.

\section{Abbreviations}

BPTF, bromodomain PHD finger transcription factor; ChIP, chromatin immunoprecipitation; GO, gene ontology; HPSE, heparanase; HS, heparan sulfate; HSPG, heparan sulfate proteoglycan; mAb, monoclonal antibody; NCR, natural cytotoxicity receptor; NK, natural killer; NURF, nucleosome remodeling factor; P+I, PMA + Ionomycin; rAd, replication-deficient adenovirus; TME, tumor microenvironment.

\section{Author contributions}

Conception and Design: K. M., Z. E., A. A., M. W., S. G. A., M. R., J. W. L.

Development and Methodology: K. M, Z. E., S. A., A. A., M. W.

Acquisition of Data: K. M, Z. E., A. A., M. W., S. G. A., M.R., C. S., K. P., V. C., N.A., P.M., T. B.

Analysis and Interpretation of Data: K. M, Z.E., A.

A., M. W., S. G. A., M. R., C. I. D., J. W. L.

Writing, review of Manuscript: K. M., Z. E., J. W. L. Administrative, Technical and Material Support: B. L. Study Supervision: C. I. D., J. W. L.

\section{ACKNOWLEDGMENTS}

The authors would like to thank members of the Michael Brown, Eric Long, Tomas Kordula labs for critically reading the manuscript. The authors would also like to thank Siddharth Saini for help packaging retrovirus.

\section{CONFLICTS OF INTEREST}

The authors have no conflicts of interest to declare.

\section{FUNDING}

These experiments were funded by start-up funds from the Department of Human and Molecular Genetics (JWL), the Massey Cancer Center (JWL), an early stage investigator grant from the $\mathrm{V}$ Foundation for Cancer Research (JWL). Services and products in support of the research project were supported with funding from $\mathrm{NIH}-$ NCI Cancer Center Support Grant P30 CA016059.

\section{REFERENCES}

1. Mittal D, Gubin MM, Schreiber RD, Smyth MJ. New insights into cancer immunoediting and its three component phases-elimination, equilibrium and escape. Curr Opin Immunol. 2014; 27:16-25.

2. Pahl J, Cerwenka A. Tricking the balance: NK cells in anticancer immunity. Immunobiology. 2017; 222:11-20.

3. Helin K, Dhanak D. Chromatin proteins and modifications as drug targets. Nature. 2013; 502:480-88.

4. Kruse PH, Matta J, Ugolini S, Vivier E. Natural cytotoxicity receptors and their ligands. Immunol Cell Biol. 2014; 92:221-29.

5. Glasner A, Ghadially H, Gur C, Stanietsky N, Tsukerman P, Enk J, Mandelboim O. Recognition and prevention of tumor metastasis by the NK receptor NKp46/NCR1. J Immunol. 2012; 188:2509-15.

6. Lakshmikanth T, Burke S, Ali TH, Kimpfler S, Ursini F, Ruggeri L, Capanni M, Umansky V, Paschen A, Sucker A, Pende D, Groh V, Biassoni R, et al. NCRs and DNAM-1 mediate NK cell recognition and lysis of human and mouse melanoma cell lines in vitro and in vivo. J Clin Invest. 2009; 119:1251-63.

7. Martner A, Rydström A, Riise RE, Aurelius J, Anderson H, Brune M, Foà R, Hellstrand K, Thorén FB. Role of natural killer cell subsets and natural cytotoxicity receptors for the outcome of immunotherapy in acute myeloid leukemia. Oncolmmunology. 2015; 5:e1041701.

8. Semeraro M, Rusakiewicz S, Minard-Colin V, Delahaye NF, Enot D, Vély F, Marabelle A, Papoular B, Piperoglou C, Ponzoni M, Perri P, Tchirkov A, Matta J, et al. Clinical impact of the NKp30/B7-H6 axis in high-risk neuroblastoma patients. Sci Transl Med. 2015; 7:283ra55.

9. Hecht ML, Rosental B, Horlacher T, Hershkovitz O, De Paz JL, Noti C, Schauer S, Porgador A, Seeberger PH. Natural cytotoxicity receptors NKp30, NKp44 and NKp46 bind to different heparan sulfate/heparin sequences. J Proteome Res. 2009; 8:712-20.

10. Xu D, Esko JD. Demystifying heparan sulfate-protein interactions. Annu Rev Biochem. 2014; 83:129-57.

11. Hammond E, Khurana A, Shridhar V, Dredge K. The Role of Heparanase and Sulfatases in the Modification of Heparan Sulfate Proteoglycans within the Tumor Microenvironment and Opportunities for Novel Cancer Therapeutics. Front Oncol. 2014; 4:195.

12. Alkhatib SG, Landry JW. The nucleosome remodeling factor. FEBS Lett. 2011; 585:3197-207.

13. Barak O, Lazzaro MA, Lane WS, Speicher DW, Picketts DJ, Shiekhattar R. Isolation of human NURF: a regulator of Engrailed gene expression. EMBO J. 2003; 22:6089-100.

14. Xiao H, Sandaltzopoulos R, Wang HM, Hamiche A, Ranallo R, Lee KM, Fu D, Wu C. Dual functions of largest NURF subunit NURF301 in nucleosome sliding and transcription factor interactions. Mol Cell. 2001; 8:531-43.

15. Qiu Z, Song C, Malakouti N, Murray D, Hariz A, Zimmerman M, Gygax D, Alhazmi A, Landry JW. Functional interactions between NURF and Ctcf regulate gene expression. Mol Cell Biol. 2015; 35:224-37. 
16. Landry J, Sharov AA, Piao Y, Sharova LV, Xiao H, Southon E, Matta J, Tessarollo L, Zhang YE, Ko MS, Kuehn MR, Yamaguchi TP, Wu C. Essential role of chromatin remodeling protein Bptf in early mouse embryos and embryonic stem cells. PLoS Genet. 2008; 4:e1000241.

17. Buganim Y, Goldstein I, Lipson D, Milyavsky M, PolakCharcon S, Mardoukh C, Solomon H, Kalo E, Madar S, Brosh R, Perelman M, Navon R, Goldfinger N, et al. A novel translocation breakpoint within the BPTF gene is associated with a pre-malignant phenotype. PLoS One. 2010; 5:e9657.

18. Aslakson CJ, Miller FR. Selective events in the metastatic process defined by analysis of the sequential dissemination of subpopulations of a mouse mammary tumor. Cancer Res. 1992; 52:1399-405.

19. Mayes K, Alkhatib SG, Peterson K, Alhazmi A, Song C, Chan V, Blevins T, Roberts M, Dumur CI, Wang XY, Landry JW. BPTF Depletion Enhances T-cell-Mediated Antitumor Immunity. Cancer Res. 2016; 76:6183-92.

20. Ogata H, Goto S, Sato K, Fujibuchi W, Bono H, Kanehisa M. KEGG: Kyoto Encyclopedia of Genes and Genomes. Nucleic Acids Res. 1999; 27:29-34.

21. Shultz LD, Lyons BL, Burzenski LM, Gott B, Chen X, Chaleff S, Kotb M, Gillies SD, King M, Mangada J, Greiner DL, Handgretinger R. Human lymphoid and myeloid cell development in NOD/LtSz-scid IL2R gamma null mice engrafted with mobilized human hemopoietic stem cells. J Immunol. 2005; 174:6477-89.

22. Marzio R, Mauël J, Betz-Corradin S. CD69 and regulation of the immune function. Immunopharmacol Immunotoxicol. 1999; 21:565-82.

23. Gong JH, Maki G, Klingemann HG. Characterization of a human cell line (NK-92) with phenotypical and functional characteristics of activated natural killer cells. Leukemia. 1994; 8:652-58.

24. Pegram HJ, Andrews DM, Smyth MJ, Darcy PK, Kershaw $\mathrm{MH}$. Activating and inhibitory receptors of natural killer cells. Immunol Cell Biol. 2011; 89:216-24.

25. Rahim MM, Makrigiannis AP. Ly49 receptors: evolution, genetic diversity, and impact on immunity. Immunol Rev. 2015; 267:137-47.

26. Brusilovsky M, Radinsky O, Cohen L, Yossef R, Shemesh A, Braiman A, Mandelboim O, Campbell KS, Porgador A. Regulation of natural cytotoxicity receptors by heparan sulfate proteoglycans in -cis: A lesson from NKp44. Eur J Immunol. 2015; 45:1180-91.

27. Brusilovsky M, Radinsky O, Yossef R, Campbell KS, Porgador A. Carbohydrate-mediated modulation of NK cell receptor function: structural and functional influences of heparan sulfate moieties expressed on NK cell surface. Front Oncol. 2014; 4:185.

28. Maki G, Klingemann HG, Martinson JA, Tam YK. Factors regulating the cytotoxic activity of the human natural killer cell line, NK-92. J Hematother Stem Cell Res. 2001; 10:369-83.
29. Wang H, Zheng X, Wei H, Tian Z, Sun R. Important role for NKp30 in synapse formation and activation of NK cells. Immunol Invest. 2012; 41:367-81.

30. Gur C, Porgador A, Elboim M, Gazit R, Mizrahi S, SternGinossar N, Achdout H, Ghadially H, Dor Y, Nir T, Doviner $\mathrm{V}$, Hershkovitz O, Mendelson M, et al. The activating receptor NKp46 is essential for the development of type 1 diabetes. Nat Immunol. 2010; 11:121-28.

31. Mandelboim O, Lieberman N, Lev M, Paul L, Arnon TI, Bushkin Y, Davis DM, Strominger JL, Yewdell JW, Porgador A. Recognition of haemagglutinins on virusinfected cells by NKp46 activates lysis by human NK cells. Nature. 2001; 409:1055-60.

32. Yossef R, Gur C, Shemesh A, Guttman O, Hadad U, Nedvetzki S, Miletić A, Nalbandyan K, Cerwenka A, Jonjic S, Mandelboim O, Porgador A. Targeting natural killer cell reactivity by employing antibody to NKp46: implications for type 1 diabetes. PLoS One. 2015; 10:e0118936.

33. Fux L, Ilan N, Sanderson RD, Vlodavsky I. Heparanase: busy at the cell surface. Trends Biochem Sci. 2009; 34:511-19.

34. Kuo $\mathrm{MH}$, Allis $\mathrm{CD}$. In vivo cross-linking and immunoprecipitation for studying dynamic Protein:DNA associations in a chromatin environment. Methods. 1999; 19:425-33.

35. Newman AM, Liu CL, Green MR, Gentles AJ, Feng W, Xu Y, Hoang CD, Diehn M, Alizadeh AA. Robust enumeration of cell subsets from tissue expression profiles. Nat Methods. $2015 ; 12: 453-57$.

36. Weinstein JN, Collisson EA, Mills GB, Shaw KR, Ozenberger BA, Ellrott K, Shmulevich I, Sander C, Stuart JM, and Cancer Genome Atlas Research Network. The Cancer Genome Atlas Pan-Cancer analysis project. Nat Genet. 2013; 45:1113-20.

37. Vendrame E, Fukuyama J, Strauss-Albee DM, Holmes S, Blish CA. Mass Cytometry Analytical Approaches Reveal Cytokine-Induced Changes in Natural Killer Cells. Cytometry B Clin Cytom. 2017; 92:57-67.

38. Zahnow CA, Topper M, Stone M, Murray-Stewart T, Li H, Baylin SB, Casero RA Jr. Inhibitors of DNA Methylation, Histone Deacetylation, and Histone Demethylation: A Perfect Combination for Cancer Therapy. Adv Cancer Res. 2016; 130:55-111.

39. Crouse J, Xu HC, Lang PA, Oxenius A. NK cells regulating $\mathrm{T}$ cell responses: mechanisms and outcome. Trends Immunol. 2015; 36:49-58.

40. Crome SQ, Nguyen LT, Lopez-Verges S, Yang SY, Martin B, Yam JY, Johnson DJ, Nie J, Pniak M, Yen PH, Milea A, Sowamber R, Katz SR, et al. A distinct innate lymphoid cell population regulates tumor-associated T cells. Nat Med. 2017; 23:368-75.

41. Ilan N, Elkin M, Vlodavsky I. Regulation, function and clinical significance of heparanase in cancer metastasis and angiogenesis. Int J Biochem Cell Biol. 2006; 38:2018-39. 
42. Ruthenburg AJ, Li H, Milne TA, Dewell S, McGinty RK, Yuen M, Ueberheide B, Dou Y, Muir TW, Patel DJ, Allis CD. Recognition of a mononucleosomal histone modification pattern by BPTF via multivalent interactions. Cell. 2011; 145:692-706.

43. Elboim M, Gazit R, Gur C, Ghadially H, Betser-Cohen G, Mandelboim O. Tumor immunoediting by NKp46. J Immunol. 2010; 184:5637-44.

44. Halfteck GG, Elboim M, Gur C, Achdout H, Ghadially H, Mandelboim O. Enhanced in vivo growth of lymphoma tumors in the absence of the NK-activating receptor NKp46/ NCR1. J Immunol. 2009; 182:2221-30.

45. Pazina T, Shemesh A, Brusilovsky M, Porgador A, Campbell KS. Regulation of the Functions of Natural Cytotoxicity Receptors by Interactions with Diverse Ligands and Alterations in Splice Variant Expression. Front Immunol. 2017; 8:369.

46. Dar AA, Nosrati M, Bezrookove V, de Semir D, Majid S, Thummala S, Sun V, Tong S, Leong SP, Minor D, Billings PR, Soroceanu L, Debs R, et al. The role of BPTF in melanoma progression and in response to BRAF-targeted therapy. J Natl Cancer Inst. 2015; 107:107.

47. Putz EM, Guillerey C, Kos K, Stannard K, Miles K, Delconte RB, Takeda K, Nicholson SE, Huntington ND, Smyth MJ. Targeting cytokine signaling checkpoint CIS activates NK cells to protect from tumor initiation and metastasis. OncoImmunology. 2017; 6:e1267892.

48. Iannello A, Thompson TW, Ardolino M, Marcus A, Raulet DH. Immunosurveillance and immunotherapy of tumors by innate immune cells. Curr Opin Immunol. 2016; 38:52-58.

49. Ribas A, Timmerman JM, Butterfield LH, Economou JS. Determinant spreading and tumor responses after peptidebased cancer immunotherapy. Trends Immunol. 2003; 24:58-61.

50. Le HK, Graham L, Cha E, Morales JK, Manjili MH, Bear HD. Gemcitabine directly inhibits myeloid derived suppressor cells in BALB/c mice bearing 4T1 mammary carcinoma and augments expansion of $\mathrm{T}$ cells from tumorbearing mice. Int Immunopharmacol. 2009; 9:900-09.

51. Urick AK, Hawk LM, Cassel MK, Mishra NK, Liu $\mathrm{S}$, Adhikari N, Zhang W, dos Santos CO, Hall JL, Pomerantz WC. Dual Screening of BPTF and Brd4
Using Protein-Observed Fluorine NMR Uncovers New Bromodomain Probe Molecules. ACS Chem Biol. 2015; 10:2246-56.

52. Galluzzi L, Buqué A, Kepp O, Zitvogel L, Kroemer G. Immunological Effects of Conventional Chemotherapy and Targeted Anticancer Agents. Cancer Cell. 2015; 28:690-714.

53. Guillerey C, Huntington ND, Smyth MJ. Targeting natural killer cells in cancer immunotherapy. Nat Immunol. 2016; 17:1025-36.

54. Luo J, Deng ZL, Luo X, Tang N, Song WX, Chen J, Sharff KA, Luu HH, Haydon RC, Kinzler KW, Vogelstein B, He TC. A protocol for rapid generation of recombinant adenoviruses using the AdEasy system. Nat Protoc. 2007; 2:1236-47.

55. Pakula R, Melchior A, Denys A, Vanpouille C, Mazurier J, Allain F. Syndecan-1/CD147 association is essential for cyclophilin B-induced activation of p44/42 mitogenactivated protein kinases and promotion of cell adhesion and chemotaxis. Glycobiology. 2007; 17:492-503.

56. Gazit R, Gruda R, Elboim M, Arnon TI, Katz G, Achdout H, Hanna J, Qimron U, Landau G, Greenbaum E, Zakay-Rones $Z$, Porgador A, Mandelboim O. Lethal influenza infection in the absence of the natural killer cell receptor gene Ncr1. Nat Immunol. 2006; 7:517-23.

57. Dumur CI, Sana S, Ladd AC, Ferreira-Gonzalez A, Wilkinson DS, Powers CN, Garrett CT. Assessing the impact of tissue devitalization time on genome-wide gene expression analysis in ovarian tumor samples. Diagn Mol Pathol. 2008; 17:200-06.

58. Singh SK, Bhardwaj R, Wilczynska KM, Dumur CI, Kordula T. A complex of nuclear factor I-X3 and STAT3 regulates astrocyte and glioma migration through the secreted glycoprotein YKL-40. J Biol Chem. 2011; 286:39893-903.

59. Huang W, Sherman BT, Lempicki RA. Systematic and integrative analysis of large gene lists using DAVID bioinformatics resources. Nat Protoc. 2009; 4:44-57.

60. Huang W, Sherman BT, Lempicki RA. Bioinformatics enrichment tools: paths toward the comprehensive functional analysis of large gene lists. Nucleic Acids Res. 2009; 37:1-13. 